\title{
AN EXTENSION OF AN INVARIANCE PROPERTY OF THE SWISS PREMIUM CALCULATION PRINCIPLE *
}

\author{
M. J. Goovaerts, F. De Vylder, F. Mertens and R. Hardy
}

\begin{abstract}
Some of the results obtained in an earlier paper entitled "An Invariance property of the Swiss premium calculation principle" by F. De Vylder and M. Goovaerts (1979) are generalized. For that purpose the notion of additivity and iterativity is extended. Some rather general characterization theorems for some premium calculation principles are obtained.
\end{abstract}

\section{INTRODUCTION}

In ref. [1] some characterization theorems for some kind of premium calculation principles are given. One of the main restrictions on the results consists in the fact that in the case of the Swiss premium calculation principle some nice properties have been deduced only in the case the parameter $z$ was a fixed constant. One considers the premium calculation principle

$$
E(v(S-z \pi))=v((1-z) \pi)
$$

for different functions $v$, but for a given constant value of $z$. During his stay at the Institute of Actuarial Sciences of the catholic university of Louvain Prof. H. Gerber suggested us to examine the case where not only different functions $v$ but also different $z$-values are considered. In the present note we formulate some rather general characterization theorems (in the framework of the Swiss premium calculation principle). Let us first recall the definition of the original Swiss premium calculation principle. Let $v$ be a continuous strictly monotonic real function defined on $=]-\infty,+\infty\left[\right.$, let further $v^{\prime}, v^{\prime \prime}$ exist in $\mathbb{R}$. Let $X$ be a real random variable. Let $\pi \in \mathbb{R}$, then we say that $\pi$ is the Swiss premium associated to the risk $X$ iff $\pi$ is the root of equation (1). Generally $\pi=\pi(X, v, z)$ depends on $X, v$ and $z$.

\section{Let further}

$\mathrm{D}_{2}$ : class of discrete random variables $X$ with strictly positive probability masses in two distinct points (but no elsewhere)

B: class of bounded random variables $X$ (those with all probability mass in a finite interval)

The function $v$ is normalized iff $v(0)=0, v^{\prime}(0)=1$ by definition.

Because $\pi(X, v, z)=\pi(X, a v+b, z)$ with $a, b$ constants and $a \neq 0$, the function $v$ occurring in a Swiss premium $\pi(X, v, z)$ can be supposed to be

* Acknowledgement: This note has benefitted from a discussion with Hans Gerber and Hans Bühlmann. 
normalized, at least when $v^{\prime} 0 \neq 0$. This last condition is always satisfied in case the original Swiss premium calculation is dealt with $\left(v^{\prime}(x)>0, v^{\prime \prime}(x) \geqslant 0\right)$. This condition is not necessarily satisfied in case the more general Swiss premium calculation principle studied in ref [1] is considered.

Let us still remark that all the statements made in the sequel will be shown to hold for $\forall X \in D_{2}$ or $X \in B$, but often immediate extensions to larger sets $D_{2}$ and $B$ are evident.

We still recall a lemma obtained in ref. [1].

\section{Lemma}

Let $z \in[0,1)$ and let $v$ be continuous strictly monotonic. Let $X \in D_{2}$ have the distribution defined as follows:

$$
p(X=a)=1-t \quad p(X=b)=t \quad(a<b, 0<t<1)
$$

Let $\pi(t)=\pi(X, v, z)$. Then $\pi(t)$ takes any value $c \in] a, b[$ when $t$ varies in ]o, $1[$. Moreover $\pi(t)$ is strictly increasing. This implies that $\pi(t)$ is continuous on $]$ o, $1[$.

\section{A SUFFICIENT CONDITION FOR AN INVARIANCE PROPERTY}

\section{Theorem 1}

Let $v_{1}, v_{2}$ be such that $v_{1}^{\prime}, v_{2}^{\prime}>0, v_{1}^{\prime \prime}, v_{2}^{\prime \prime}$ exist and let $z_{1}, z_{2} \in[0,1[$. Then:

$$
\pi\left(X, v_{1}, z_{1}\right)=\pi\left(X, v_{2}, z_{2}\right)
$$

implies:

$$
v_{1}^{\prime}\left(x\left(1-z_{1}\right)\right)^{1-z_{2}}=c v_{2}^{\prime}\left(x\left(1-z_{2}\right)\right)^{1-z_{1}}
$$

where $c$ is some constant independent of $x \in \mathbb{R}$.

\section{Demonstration}

Let

$$
F_{X}(x)=(1-t) H(x-a)+t H(x-b) \quad a<b
$$

where $H()$ denotes as usual the Heaviside function.

$\pi=\pi\left(X, v_{1}, z_{1}\right)=\pi\left(X, v_{2}, z_{2}\right)$ satisfies the following set of equations:

$$
\begin{aligned}
& (1-t) v_{1}\left(a-z_{1} \pi\right)+t v_{1}\left(b-z_{1} \pi\right)=v_{1}((1-z) \pi) \\
& (1-t) v_{2}\left(b-z_{2} \pi\right)+t v_{2}\left(b-z_{2} \pi\right)=v_{2}\left(\left(1-z_{2}\right) \pi\right) .
\end{aligned}
$$

These equations can still be cast into the form:

$$
\frac{v_{1}\left(\left(1-z_{1}\right) \pi\right)-v_{1}\left(b-z_{1} \pi\right)}{v_{1}\left(a-z_{1} \pi\right)-v_{1}\left(\left(1-z_{1}\right) \pi\right)}=\frac{1-t}{t}
$$


(7)

$$
\frac{v_{2}\left(\left(1-z_{2}\right) \pi\right)-v_{2}\left(b-z_{2} \pi\right)}{v_{2}\left(a-z_{2} \pi\right)-v_{2}\left(\left(1-z_{2}\right) \pi\right)}=\frac{1-t}{t}
$$

Elimination of $t$ gives:

$$
\frac{v_{1}\left(\left(1-z_{1}\right) \pi\right)-v_{1}\left(b-z_{1} \pi\right)}{v_{1}\left(a-z_{1} \pi\right)-v_{1}\left(\left(1-z_{1}\right) \pi\right)}=\frac{v_{2}\left(\left(1-z_{2}\right) \pi\right)-v_{2}\left(b-z_{2} \pi\right)}{v_{2}\left(a-z_{2} \pi\right)-v_{2}\left(\left(1-z_{2}\right) \pi\right)}
$$

which hold for $a<b, \forall t \in] \quad 0,1]$.

Because one is able (according to the lemma of paragraph 1) to solve the system of equations (6) with respect to $t$ for each given $\pi \in$ ] $a, b$ [we conclude (8) to hold for every $a, b$, $\pi$ for which $a \leqslant \pi \leqslant b$.

Now $(8)$ can be cast into the form :

(9)

$$
\left|\begin{array}{ccc}
1 & 1 & 1 \\
v_{1}\left(a-z_{1} \pi\right) & v_{1}\left(\left(1-z_{1}\right) \pi\right) & v_{1}\left(b-z_{1} \pi\right) \\
v_{2}\left(a-z_{2} \pi\right) & v_{2}\left(\left(1-z_{2}\right) \pi\right) & v_{2}\left(b-z_{2} \pi\right)
\end{array}\right|=0
$$

$\forall a, b, \pi$ (with $a \leqslant \pi \leqslant b$ )

The condition (9) can still be cast into the form

$$
\begin{aligned}
& \left|\begin{array}{ccc}
1 & 0 & 0 \\
v_{1}\left(a-z_{1} \pi\right) \frac{v_{1}\left(\left(1-z_{1}\right) \pi\right)-v_{1}\left(a-z_{1} \pi\right)}{\pi-a} & \frac{v_{1}\left(b-z_{1} \pi\right)-2 v_{1}\left(\left(1-z_{1}\right) \pi\right)+v_{1}\left(a-z_{1} \pi\right)}{(\pi-a)(\pi-b)} \\
v_{2}\left(a-z_{2} \pi\right) \frac{v_{2}\left(\left(1-z_{2}\right) \pi\right)-v_{2}\left(a-z_{2} \pi\right)}{\pi-a} & \frac{v_{2}\left(b-z_{2} \pi\right)-2 v_{2}\left(\left(1-z_{2}\right) \pi\right)+v_{2}\left(b-z_{2} \pi\right)}{(\pi-a)(\pi-b)}
\end{array}\right| \\
& =0
\end{aligned}
$$

Taking the limit for $\pi \rightarrow a$ and $b \rightarrow \pi \rightarrow a$ the following result is obtained:

$$
v_{1}^{\prime}\left(x-z_{1} x\right) v_{2}^{\prime \prime}\left(x-z_{2} x\right)=v_{1}^{\prime \prime}\left(x-z_{1} x\right) v_{2}^{\prime}\left(x-z_{2} x\right)
$$

$\forall x \in \mathbb{R}$

Hence performing one integration with respect to $x(11)$ is transformed into (4).

\section{Remarks}

- If only positive risks are considered $(a, b>0)$ (4) holds only $\forall x \in \mathbb{R}^{+}$.

- The condition (4) is transformed into

$$
v_{1}^{\prime}\left(x\left(1-z_{1}\right)\right)^{1-z_{2}}=v_{2}^{\prime}\left(x\left(1-z_{2}\right)\right)^{1-z_{1}}
$$

in case one considers normalized $v_{1}$ and $v_{2}$. 
148 M. J. GOOVAERTS, F. DE VYLDER, F. MERTENS AND R. HARDY

- In fact condition (4) is not a sufficient condition as can be seen from the following counter example

Indeed: let $v_{1}(x)=x^{3}$ and $z_{1}=0,5$

$$
v_{2}(x)=x^{5} \text { and } z_{2}=0
$$

and consider

$$
F_{X}(x)=(1-t) H(x)+t H(x-1)
$$

Clearly $v_{1}(x), v_{2}(x)$ satisfy $(4)$ but the corresponding premiums are different in case $t \neq 1$

3. A NECESSARY AND SUFFICIENT CONDITION FOR THE INVARIANCE PROPERTY

Theorem 2

Let $o \leqslant z_{1} \leqslant z_{2} \leqslant 1$. Suppose that $v_{1}, v_{2}$ are normalized, have continuous positive derivatives and that $v_{1}^{\prime \prime}(\mathrm{o})$ exists.

If

$$
\pi\left(X, v_{1}, z_{1}\right)=\pi\left(X, v_{2}, z_{2}\right)
$$

for all $X \in D_{2}$ then exactly one of the following situations occurs:

i) $z_{1}=z_{2}, v_{1}=v_{2}$

ii) $z_{1} \neq z_{2}$ and for some $c \neq 0, v_{1}(x)=v_{2}(x)=\frac{1}{c}\left(e^{c x}-1\right) \quad(x \in \mathbb{R})$

iii) $z_{1} \neq z_{2}, v_{1}(x)=v_{2}(x)=x \quad(x \in \mathbb{R})$

Conversely, each of (i), (ii), (iii) imply that $\pi\left(X, v_{1}, z_{1}\right)=\pi\left(X, v_{2}, z_{2}\right)$ for all $X \in B$.

\section{Demonstration}

The converse part is evident. We only have to prove the direct part of the theorem. We admit (12) for all $X \in D_{2}$.

j) Here we prove that $v_{1}=v_{2}$. Indeed fro $m(12)$ results the implication valid for all $X \in D_{2}, \pi \in \mathbb{R}$ :

$$
E\left(v_{1}\left(X-x_{1} \pi\right)\right)=v_{1}\left(\left(1-z_{1}\right) \pi\right) \Rightarrow E\left(v_{2}\left(X-z_{2} \pi\right)\right)=v_{2}\left(\left(1-z_{2}\right) \pi\right)
$$

In particular, for $\pi=0: E\left(v_{1}(X)\right)=0 \Rightarrow E\left(v_{2}(X)\right)=0$

For $X$ distributed as follows:

$$
p(X=x)=t_{0}, p(X=a)=1-t_{0}\left(x \neq a, 0<t_{0}<1\right)
$$

where $t_{0}$ is choosen such that $\pi\left(t_{0}\right)=0$ according to lemma 1 we obtain:

$$
t_{0} v_{1}(x)+\left(1-t_{0}\right) v_{1}(a)=0 t_{0} v_{2}(x)+\left(1-t_{0}\right) v_{2}(a)=0 .
$$


Taking $a=1$, we see that we must have $v_{2}(x)=b v_{1}(x)$ for some constant $b$ and all $x<0$. Similarly, for $a=-1$, we conclude that $v_{2}(x)=c v_{1}(x)$ for some $c$ and all $x<0$. Taking left and right derivatives at the origine we find that $b=$ $c=1$.

If $z_{1}=z_{2}$, we are in case (i) and we have finished. Therefore we may assume $z_{1}<z_{2}$ from now on. We also write $v=v_{1}=v_{2}$.

jj) Now we prove the following:

For each $X \in D_{2}$ there is a $\left.z_{0} \in\right] z_{1}, z_{2}\left[\right.$ and $a \pi_{0} \in \mathbb{R}$ satisfying

and

$$
E\left(v\left(X-z_{0} \pi_{0}\right)\right)=v\left(\left(1-z_{0}\right) \pi_{0}\right)
$$

(13) where

$$
\begin{gathered}
\pi_{0} E\left(v_{0}\left(X-z_{0} \pi_{0}\right)\right)=\pi_{0} v_{0}\left(\left(1-z_{0}\right) \pi_{0}\right), \\
v_{0}(x)=v^{\prime}(x)-v^{\prime}(0),(x \in \mathbb{R})
\end{gathered}
$$

Indeed, let $X \in D_{2}$ be fixed. For $0 \leqslant z \leqslant 1$ let $\pi(z)$ be defined by

$$
E(v(X-z \pi(z)))=v((1-z) \pi(z))
$$

By an adequate implificitfunctions theorem $\pi(z)$ has a continuous derivative.

Derivation of the last identity gives:

$$
-\left(\pi(z)-z \pi^{\prime}(z)\right) E\left(v^{\prime}(X-z \pi(z))=\left(\pi^{\prime}(z)-\pi(z)-z \pi^{\prime}(z)\right) v^{\prime}((1-z) \pi(z)) .\right.
$$

The assumptions imply that $\pi\left(z_{1}\right)=\pi\left(z_{2}\right)$ and therefore there is a $\left.z_{0} \in\right] z_{1}, z_{2}[$ such that $\pi^{\prime}\left(z_{0}\right)=0$. Then (13) follows for $\pi_{0}=\pi\left(z_{0}\right)$.

jij) The next step is to prove that the factor $\pi_{0}$ can be dropped in each member of the last relation in (13) even when $\pi_{0}=0$. Indeed, the first relation in (13) shows that $\pi_{0}=0$ is equivalent to $E(v(X))=0$. Then consider the variables $X_{n}=X+\frac{1}{n}$ and the corresponding $z_{n}, \pi_{n}(n=1,2 \ldots)$. It is evident that $\pi_{n}>\pi_{0}=0$. Going over to subsequences, we may assume that $z_{n} \rightarrow z, \pi_{n} \rightarrow \pi$. Considering (13) for $X_{n}, \pi_{n}, z_{n}$, simplifying by $\pi_{n}$ in the last relation and letting $n \rightarrow \infty$, we obtain $\pi=0$ and $E\left(v_{0}(X)\right)=0$.

jijj) In fact we proved that: $E(v(X))=0 \Rightarrow E\left(v_{0}(X)\right)=0$. Then the argument in $j$ ) shows that $v_{0}=a v$ for some constant $a$. Remembering the definition of $v_{0}$ and the normalization conditions, this implies that we must be in case (ii) or (iii).

\section{Remarks}

- The conditions on the functions $v_{1}, v_{2}$ could be weakened.

- The theorem 2 has probably a version for nonnegative risks only.

If this is the case, the corresponding demonstration must proceed along different lines. 


\section{APPLICATION: GENERALIZED ADDITIVITY}

\section{Definition}

The set $\left\{\left(v_{1}, z_{1}\right),\left(v_{2}, z_{2}\right),(v, z)\right\}$ is said to generate additive premium calculation principles in the generalized sense if:

$$
\pi(X+Y, v, z)=\pi\left(X, v_{1}, z_{1}\right)+\pi\left(Y, v_{2}, z_{2}\right)
$$

whatever be the independent random variables $X, Y \in B$.

\section{Theorem 3}

The set $\left\{\left(v_{1}, z_{1}\right),\left(v_{2}, z_{2}\right),(v, z)\right\}$ generates an additive premium calculation principle in the generalized sense if $\left(v_{1}, z_{1}\right),\left(v_{2}, z_{2}\right),(v, z)$ give raise to the same exponential premium calculation principle (containing the expected value principle as limiting case).

\section{Demonstration}

Let $X \in D_{2}$ and hence:

$$
v_{1}\left(\left(1-z_{1}\right) \pi_{1}\right)=t v_{1}\left(a-z_{1} \pi_{1}\right)+(1-t) v_{1}\left(b-z_{1} \pi_{1}\right)
$$

where we used the abbreviation $\pi_{1}=\pi\left(X, v_{1}, z_{1}\right)$.

Let $Y$ be the random variable with its total probability mass 1 placed in the point $y$ and hence:

$$
v_{2}\left(\left(1-z_{2}\right) \pi_{2}\right)=v_{2}\left(y-z_{2} \pi_{2}\right)
$$

where $\pi_{2}=\pi\left(Y, v_{2}, z_{2}\right)=y$

Because of the additivity property $\pi=\pi_{1}+y$ is the root of

$$
v((1-z) \pi)=t v(a+y-z \pi)+(1-t) v(b+y-z \pi)
$$

Putting $v(x+(1-z) y)=w(x)$ transforms (15) into

$$
w\left((1-z) \pi_{1}\right)=t w\left(a-z \pi_{1}\right)+(1-t) x\left(b-z \pi_{1}\right)
$$

Comparing (13) and (16) the following result is obtained according to our theorem in section 2 :

$$
v_{1}^{\prime}\left(x\left(1-z_{1}\right)\right)^{1-z}=c w^{\prime}(x(1-z))^{1-z_{1}}
$$

Or:

$$
v_{1}^{\prime}\left(x\left(1-z_{1}\right)\right)^{1-z}=c v^{\prime}(x(1-z)+y(1-z))^{1-z_{1}} .
$$

No restriction is imposed assuming $v_{1}^{\prime}(0)=1$. Hence:

$$
v^{\prime}(x(1-z)+y(1-z))^{1-z_{1}}=v_{1}\left(x\left(1-z_{1}\right)\right)^{1-z} \cdot v^{\prime}(y(1-z))^{1-z_{1}}
$$


Consequently:

$$
\begin{array}{ll}
v^{\prime}(x)=e^{\alpha x} & \alpha \geqslant 0 \\
v_{1}(x)=e^{\alpha x} & \alpha \geqslant 0
\end{array}
$$

\section{Remarks}

- The demonstration of theorem 3 only holds in case risks with an arbitrary sign are considered.

Indeed in case only positive risks are concerned the relation (17) only holds in case $x \in \mathbb{R}^{+}$.

- The following additional remarks however show that the theorem 3 also holds in case only positive risks are considered.

Indeed the demonstration of theorem 3 shows that for the present case

$$
v(x)=v_{1}(x)=v_{2}(x)=e^{\alpha x} \quad \forall x \in \mathbb{R}^{+} .
$$

However (13) and (14) yields:

$$
\left\{\begin{array}{l}
e^{\alpha(1-z) \pi}=e^{-\alpha(1-z) y} v(-z \pi+(1-z) y)+(1-t) e^{\alpha(b-z \pi)} \\
e^{\alpha(1-z) \pi}=v_{1}(-z \pi)+(1-t) e^{\alpha(b-z \pi)}
\end{array}\right.
$$

having put $a=0$.

Consequently:

$$
v_{1}(-z \pi)=e^{\alpha(1-z) y} v(-z \pi+(1-z) y)
$$

valid for all positive $\pi$ and $y$.

This equation extends $v(x)$ and $v_{1}(x)$ to the case where $x \in \mathbb{R}^{-}$. Clearly this implies that both $v_{1}(x)$ and $v(x)$ are of the desired exponential form.

\section{APPLICATION: GENERALIZED ITERATIVITY}

\section{Definition}

The set $\left\{\left(v_{1}, z_{1}\right),\left(v_{2}, z_{2}\right),(v, z)\right\}$ is said to generate iterative premium calculation principles in the generalized sense if for each $X, Y \in B$ the relations:

$$
\begin{gathered}
E\left(v_{1}\left(X-z_{1} \pi\left(X / Y, v_{1}, z_{1}\right)\right) / Y\right)=v_{1}\left(\left(1-z_{1}\right) \pi\left(X / Y, v_{1}, z_{1}\right)\right) \\
E\left(v_{2}\left(\pi\left(X / Y, v_{1}, z_{1}\right)-z_{2} \pi^{*}\right)=v_{2}\left(\left(1-z_{2}\right) \pi^{*}\right)\right. \\
E(v(X-z \pi))=v((1-z) \pi) \\
\pi^{*}=\pi
\end{gathered}
$$

(23) imply:

\section{Theorem 4}

Let $v_{1}^{\prime}, v_{2}^{\prime}, v^{\prime}>0 ; v_{1}^{\prime \prime}, v_{2}^{\prime \prime}, v^{\prime \prime} \geqslant 0$ exist. Let $\left.z_{1}, z_{2}, z \in\right] 0,1[$. 
Then the set $\left\{\left(v_{1}, z_{1}\right),\left(v_{2}, z_{2}\right),(v, z)\right\}$ is iterative in the generalized sense if

$$
v_{1}(x)=v_{2}(x)=v(x)=\alpha(x)
$$

where $\alpha(x)$ is a lineair function of $x$ or of $e^{\alpha x}$ with a $>0$.

Remark:

This result will shown to hold as soon as $z_{2} \neq 0$. It seems to be rather difficult to show that in case $\left(v_{2}, z_{2}\right)$ reduces to a mean value principle one should have also $0=z_{1}=z$ and $v_{2}(x)=v_{1}(x)=v(x)$.

\section{Demonstration}

Let us consider :

$$
F_{Y / X}(x)=y H(x-a)+(1-y) H(x-b)
$$

$X / Y$ thus denotes a Bernouilli risk

$$
\begin{gathered}
p(X / Y=a)=y \\
p(X / Y=b)=1-y
\end{gathered}
$$

Let $\pi(y)=\pi\left(X / Y, v_{1}, z_{1}\right)$ then:

$$
v_{1}\left(\left(1-z_{1}\right) \pi(y)\right)=y v_{1}\left(a-z_{1} \pi(y)\right)+(1-y) v_{1}\left(b-z_{1} \pi(y)\right)
$$

On the other hand one has:

$$
F_{X}(x)=m H(x-a)+(1-m) H(x-b) \text { where } m=E(Y)
$$

such that the collective premium is obtained by solving

$$
v((1-z) \pi(m))=m v(a-z \pi(m))+(1-m) v(b z-\pi(m))
$$

where $\pi(m)=\pi(X, v, z)$.

Iteratively imply that this premium $\pi(m)$ also is the root of the equation determing the iterative premium:

$$
v_{2}\left(\left(1-z_{2}\right) \pi(m)\right)=\int_{0}^{1} v_{2}\left(\pi(y)-z_{2} \pi(m)\right) d F_{Y}(y)
$$

This last equation can be cast into the form:

$$
\int_{0}^{1}\left[v_{2}\left(\pi(y)-z_{2} \pi(m)\right)-v_{2}\left(\left(1-z_{2}\right) \pi(m)\right)\right] d F_{Y}(y)=0
$$

together with the conditions:

$$
\begin{aligned}
& \int_{0}^{1} d F_{Y}(y)=1 \\
& \int_{0}^{1} y d F_{Y}(y)=m
\end{aligned}
$$


which imply that:

$$
v_{1}\left(\pi(y)-z_{2} \pi(m)\right)-v_{2}\left(\left(1-z_{2}\right) \pi(m)\right)-\alpha y-\beta=0
$$

where $\alpha$ and $\beta$ are certain constants (independent of $y$ ). As has been shown in a previous paper [2], (33) can be transformed into:

$$
v_{2}\left(\pi(y)-z_{2} \pi(m)\right)=y v_{2}\left(a-z_{2} \pi(m)\right)+(1-y) v_{2}\left(b-z_{2} \pi(m)\right)
$$

In case $z_{2} \neq 0$ we can proceed in much the same way as in ref. [2] to conclude that $v_{2}(x)$ has to be the exponential or a linear function.

But $v_{2}$ being linear or exponential (34) can be cast into the form:

$$
v_{2}\left(\pi(y)-z_{2} \pi(y)\right)=y v_{2}\left(a-z_{2} \pi(y)\right)+(1-y) v_{2}\left(b-z_{2} \pi(y)\right)
$$

Comparing (28) with (35) allows us to apply theorem 1 of section 2 , such that

$$
v^{\prime}(x(1-z))^{1-z_{2}}=c v_{2}^{\prime}\left(x\left(1-z_{2}\right)\right)^{1-z}
$$

which implies that also $v(x)$ denotes an exponential or a linear function.

Of course (35) can also be compared with (26) and consequently another application of theorem 1 provides the desired result: the normalized $v_{1}(x)$, $v_{2}(x)$ and $v(x)$ coincide. The proof of the converse is elementary.

\section{REFERENCES}

De Vylder, F. and M. J. Goovaerts. (1979). An invariance property of the Swiss premium calculation principle. M.V.S.V., 79, 105-120.

Goovaerts, M. J. and F. De Vylder. (1979). A note on iterative premium calculation principles. Astin Bulletin 10, 326-329. 